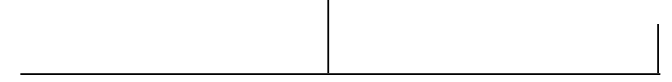

Rev. Latinoam. Psicopat. Fund., São Paulo, v. 11, n. 1, p. 105-118, março 2008

\title{
Dinâmica não-linear e psiquiatria: a natureza dinâmica das doenças mentais
}

\author{
Fernando Portela Câmara
}

A psiquiatria moderna adotou a postura reducionista de associar psicopatologia à fisiologia sináptica. Isto produziu avanços importantes na farmacoterapia dos transtornos mentais. Entretanto, a introdução de princípios da dinâmica não-linear promoveu uma mudança do enfoque sináptico para o enfoque das vias específicas patologicamente autônomas envolvidas nos fenômenos psicopatológicos. Esta tendência teve início nos anos 1990 e trouxe um conceito mais sistêmico de fisiopatologia e tratamento em psiquiatria.

Palavras-chave: Psiquiatria e dinâmica não-linear, termodinâmica do não-equilíbrio e psiquiatria, doenças dinâmicas 
... The present era of qualitative mathematical description of the behaviour of complex (...) systems promises a language for a scientifically based (...) fusion of clinical psychiatric phenomenology and neurobiological mechanism.

(Mandell, 1982)

O uso transdisciplinar da dinâmica não-linear (popularmente conhecida como teoria do caos) e da teoria da complexidade abriu uma nova perspectiva de análise em medicina (Ebert et al., 1994). Uma grande massa de dados, acumulada em muitos anos de pesquisa, pôde ser analisada dentro de um novo marco conceitual (Skarda e Freeman, 1990; Mandell e Selz, 1992) sem transpor inadequadamente os limites conceituais que garantem a realidade dos modelos propostos. Os anos 1990 foram especialmente férteis para a psiquiatria, pois a incorporação de paradigmas da dinâmica não-linear (Freeman, 1992; Mandell e Selz, 1992) modificou, de modo ainda não totalmente percebido pela maioria dos psiquiatras, o paradigma da fisiologia das sinapses para a fisiologia das vias implicadas nos sintomas, atual enfoque das pesquisas psicofarmacológicas.

Uma das contribuições deste ramo novo da ciência à medicina foi o conceito de enfermidade dinâmica (Mackey e Milton, 1987; Goldberger, 1990, 1991). Esses e outros autores demonstraram objetivamente como a fisiologia de determinados processos pode se modificar dinamicamente levando a doenças graves (ver, por exemplo, Redington e Reidbord, 1992; Mandell, 1982; Pool, 1989; Pezard et al., 1996; Schiff et al., 1994; Yeragami et al., 2002; Paulus e Braff, 2002). Também é possível estabelecer modelos razoáveis para doenças mentais, onde falta a demonstração de alterações materiais ou marcadores biológicos (sine materiae), e cuja complexidade fisiológica do cérebro não nos permite ainda obter um conhecimento suficiente para inferir sobre mecanismos. Entretanto, tais modelos nos permitem insights teóricos e práticos sobre origem e tratamento destas doenças.

O paradigma vigente no modelo médico tradicional ainda é o da causa específica, estabelecido a partir dos experimentos de Pasteur (teoria dos germes) e Virchow (teoria da lesão), no século XIX. Este paradigma ganharia reforço com a teoria da "bala mágica", formulada por Erlich no começo do século XX, dando início à quimioterapia e antibioticoterapia. Estes foram os fundamentos que originaram e impulsionam a pesquisa farmacológica na medicina, culminando com a atual parceria da indústria. 
A idéia de enfermidade dinâmica remonta a essa época, defendida por Claude Bernard, que se opunha à teoria da causa específica em inflamados debates com Pasteur, defendendo que não era o micróbio a causa da doença, mas o terreno, o estado do organismo ("nas infecções o terreno é tudo, o micróbio não é nada"). Ele partia da observação de que nem todos os indivíduos adoecem quando expostos ao mesmo germe ou ao mesmo fator morbígeno. Para explicar sua tese, criou o conceito de "homeostase", definida como a capacidade do organismo de manter a constância dos seus parâmetros fisiológicos. Apesar desta corrente de pensamento ter sido formalizada na Teoria Geral dos Sistemas (Von Bertalanffy, 1975), sua abordagem sistêmica não conseguiu estabelecer modelos cientificamente aceitáveis para a perturbação da homeostase como causa essencial das enfermidades. Esta tarefa caberia ao desenvolvimento mais recente da dinâmica não-linear.

A idéia de homeostase, preconizada por Bernard, baseava-se na noção platônica de equilíbrio, harmonia. Contudo, sabemos hoje que a homeostase dos organismos é dinâmica, ou melhor, adaptativa. Por exemplo, a produção de eritropoietina aumenta quando saímos do nível do mar e vamos para lugares de altitude elevada, onde o oxigênio é rarefeito e precisamos de mais hemácias, e retorna aos níveis originais se voltarmos ao lugar anterior. Claramente se percebe que o conceito de homeostase não pode estar associado à noção de equilíbrio estacionário, mas a uma condição dinâmica, flexível, adaptativa, integrada. A compreensão deste tipo de homeostase foi possível a partir da dinâmica nãolinear, que nos mostra como um sistema pode ter diferentes dinâmicas quando se modifica seus parâmetros de controle. É neste sentido que King e Liston (1990) e Pires (1981) concebem a homeostase ao proporem uma "homeostase patológica" ou um "desmantelamento da homeostase", respectivamente, ao mesmo fenômeno estudado por Goldberger (1990) como "enfermidade dinâmica".

Uma analogia pode fazer melhor entender esta homeostase que prefiro chamar de homeostase adaptativa. A aeronave de combate B-2 Spirit marcou uma revolução tecnológica (tecnologia "Stealth", por ser "invisível" aos radares inimigos). Aerodinamicamente, esta aeronave não é estável e não pode voar, por isso é equipada com 168 computadores que calculam, a cada minuto, as variações de numerosos parâmetros corrigindo-os por feedback, tornando o vôo possível e, mais que isso, preciso, seguro e altamente eficiente. A complexidade desta aeronave é de tal ordem que sua pilotagem humana é impossível, pois um ser humano não consegue detectar oscilações mínimas e, ao mesmo tempo, operar milhares de ajustes para corrigi-las. Esta complexidade dinâmica torna o B-2 uma aeronave versátil e adaptativa, analogamente a um organismo.

Pavlov foi o primeiro a propor objetivamente que alterações na dinâmica dos processos de excitação e inibição seriam o substrato dos processos 
psicopatológicos. Ele considerava a origem das doenças mentais e somáticas como uma dificuldade no processo de adaptação que resultava de uma perturbação na dialética contínua entre aqueles processos antagônicos. Numa outra situação, Freud postulou uma origem psíquica para as mesmas, mas não soube explicar fisiologicamente como seu modelo podia organizar os sintomas e sua evolução, assentando sua doutrina numa hipótese idealista do inconsciente; hoje, todavia, a dinâmica não-linear pode fornecer um insight fisiológico para sua teoria.

\section{Bifurcações, estruturas dissipativas e ordem por flutuação}

Os sistemas complexos, isto é, sistemas formados por um grande número de componentes interagindo entre si, são caracteristicamente instáveis e governados por uma dinâmica não-linear. São sistemas abertos, em cuja fronteira ocorrem trocas contínuas de matéria, energia e informação com o ambiente. A física clássica trata tais fluxos como uma situação idealizada em que o sistema encontra-se em equilíbrio e comporta-se de forma homogênea. Toda perturbação que não seja demasiadamente intensa tende a afastar o sistema deste ponto estável (chamado de ponto atrator), mas a ele retorna quando a perturbação cessa. Este comportamento previsível é descrito em aproximações lineares. No mundo real, estes sistemas estão sujeitos a constantes flutuações locais, de modo que eles não estão exatamente em equilíbrio, e sim oscilando em torno deste, e ainda mantendo sua homogeneidade. A parte da física que estuda estes sistemas é conhecida como termodinâmica de sistemas afastados do equilíbrio, e foi fundada por Ilya Prigogine e colaboradores.

Estas flutuações surgem e desaparecem, porém, ocasionalmente, uma flutuação local pode amplificar-se por efeito de um feedback positivo, levando o sistema a se afastar mais e mais do seu equilíbrio original até atingir um limiar ou valor crítico onde perderá toda estabilidade. Neste ponto, chamado ponto de bifurcação, o sistema muda subitamente em qualidade, perde sua homogeneidade ("quebra de simetria"), exibindo um novo tipo de ordem, e adquire propriedades novas. A instabilidade atingida redireciona o sistema para uma outra condição metaestável agora mais complexa. Essas bifurcações caracterizam um sistema nãolinear, isto é, que muda dinamicamente quando experimenta uma variação mínima numa região crítica. A figura 1 ilustra este princípio de não-linearidade.

Se a amplificação da flutuação não é suficiente para o sistema alcançar um ponto de bifurcação, ele tenderá a se restaurar; porém, se este ponto é atingido, ele muda subitamente e de modo irreversível, mas é necessário que a energia que "empurrou" o sistema para esta nova condição seja mantida, senão ele se 


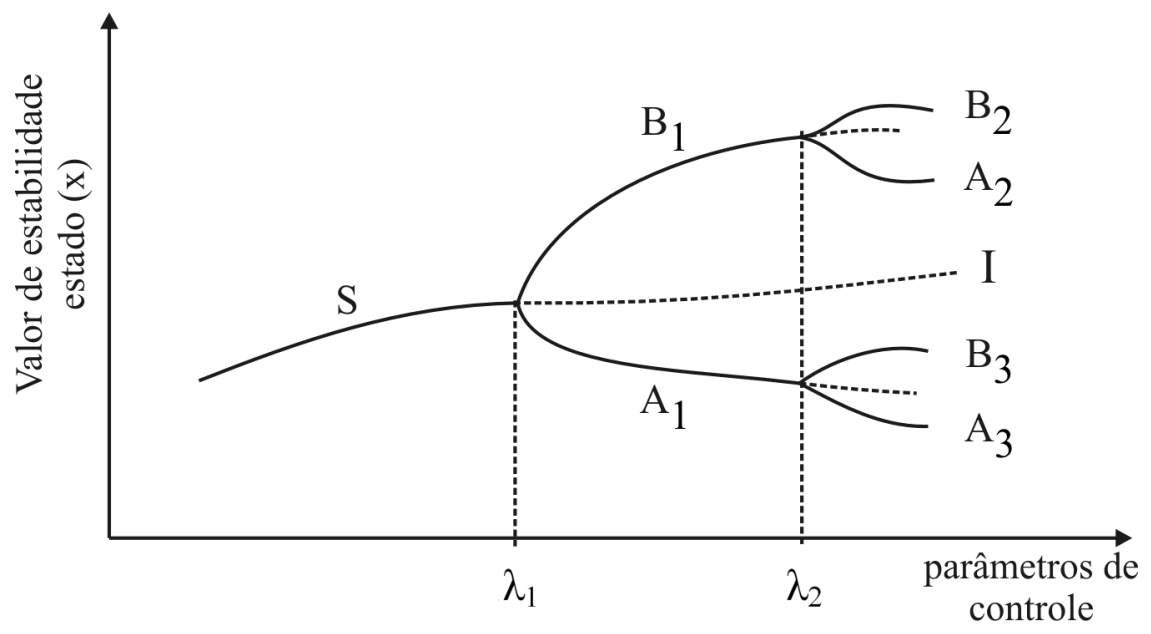

Figura 1 - Mapa de uma dinâmica não-linear em função do parâmetro de controle do sistema. $\lambda$ é o parâmetro de controle; $\lambda_{1}$ e $\lambda_{2}$ são os valores críticos (ou pontos de bifurcação) em que o sistema torna-se instável e subitamente e novos valores de estabilidade (A ou $B$ ) emergem. As linhas sólidas representam os valores estáveis (equilíbrio estacionário) e as pontilhadas os valores instáveis, proibitivos. A escolha de uma via para qualquer das soluções está sujeita a flutuações, portanto, é probabilística e irreversível.

desintegra e volta à condição homogênea, randômica, do equilíbrio original. Por tal razão, Prigogine (1984a) chamou este fenômeno de "ordem por flutuação", e como esta nova ordem decorre somente desta dinâmica, sendo espontânea e sem informação prévia para originá-la, o processo é também conhecido como "autoorganização". Em dialética, isto se denomina "salto de qualidade" e, em física, "ruptura de simetria" (homogeneidade original), pois o sistema passa a manifestar um modo de funcionamento coerente e qualitativamente novo, que se distingue e compete com aquele resto que não sofreu flutuação.

Deste modo, à medida que um sistema complexo afasta-se do equilíbrio, ele experimenta auto-organização, que se torna mais complexa à medida que este afastamento aumenta e novos pontos de bifurcação são alcançados, até que, subitamente, perca toda ordem ao atingir o caos. O sistema alcança maior ordem (e maior instabilidade) na região limite do caos.

Como já dissemos, a ordem é mantida pela flutuação que lhe deu origem, desde que o feedback positivo ou aporte de energia que a sustenta sejam mantidos. Isto é necessário para manter a nova ordem (a nova estrutura ou função) ao 
mesmo tempo que libera entropia para o ambiente. A ordem deve, portanto, ser regenerada continuamente ao mesmo tempo em que se desintegra continuamente, pois é num ciclo incessante de criação e destruição que os seres passam a existir, "a luta constante entre ordem e desordem" como Empédocles, Anaximandro e Heráclito concebiam a Natureza. Prigogine chama estas ordens de "estruturas dissipativas" ou "zonas de flutuação" (atratores caóticos). A luta entre a zona flutuante e o resto do sistema, que não sofreu flutuação, é constante, esta tende a invadir aquela e aquela tende a neutralizar esta.

O leitor poderá entender mais ainda ao ler a próxima seção.

\section{Instabilidade de Rayleigh-Bénard}

Se colocarmos uma película de um líquido entre duas placas de vidro e aquecermos ligeiramente a placa inferior, a agitação térmica será distribuída igualmente por toda massa líquida, equilibrando-se. Qualquer flutuação (perturbações mínimas) no sistema será extinta e ele permanecerá no seu estado original. Esta ordem homogênea e internamente aleatória chama-se "ordem de Boltzman". Se continuarmos a elevar gradual e controladamente a temperatura (isto simula um feedback positivo), veremos que, quando a temperatura atinge um valor crítico (ponto de bifurcação), o sistema se torna subitamente instável e perde sua homogeneidade, aparecendo rolos de convecção onde antes era um fluido homogêneo. A flutuação térmica, amplificada para além de um valor crítico, domina agora todo o sistema, empurrando-o cada vez mais para longe do equilíbrio original. Esta bifurcação leva o sistema a adquirir um padrão de forma (no caso, rolos de convecção), e isso de modo espontâneo, sem instrução ou informação prévia (isto é, impossível de se prever a partir dos componentes do sistema), fenômeno conhecido como "auto-organização" ou "ordem por flutuação". O sistema mantém sua nova ordem na medida em que o aporte de energia que o gerou é mantido, ao mesmo tempo em que ele dissipa a entropia destruindo-se e regenerando-se continuamente, daí sua instabilidade. Os rolos de convecção de célula, de Bénard, é um padrão de forma mantido pela cooperação de todas as moléculas envolvidas ("correlação"), que antes se distribuíam aleatoriamente (movimento Browniano). Tais são as propriedades de uma estrutura dissipativa, cuja ordem só é possível quando o sistema se afasta do equilíbrio, tornando-se transiente e, portanto, instável. Os processos biológicos e, por extensão, da natureza não existem no equilíbrio estacionário, como idealizavam a física e a química clássicas, mas se mantêm permanentemente afastados desta condição (Prigogine, 1984b). 
Se continuarmos aumentando a temperatura da placa inferior, atingiremos valores críticos onde mais instabilidade será gerada, e mais longe do equilíbrio se encontrará o sistema. Os rolos se duplicarão até chegar num valor onde o sistema se tornará turbulento, portanto, caótico. Será na região próxima do ponto de turbulência (caos) que a estrutura dissipativa se tornará mais complexa.

\section{O cérebro em fluxo}

O processamento da informação cerebral é um sistema dialético envolvendo o córtex e a região subcortical, questão já aventada por Pavlov, Bykov e outros, que situavam a sede dos processos dialéticos entre cérebro/organismo/ambiente no eixo cortico-subcortical. Este eixo é o centro da integração organísmica, caracterizando-se como um sistema complexo em permanente flutuação e cuja instabilidade permite-lhe interagir com o meio, adaptando-se constantemente a ele. Este sistema é regulado em todos os níveis por feedback. Perturbações locais podem ser amplificadas por feedbacks positivos e logo contrabalançadas por feedbacks negativos (regulatórios), do contrário, poderá ocorrer uma ruptura funcional local. O risco desta disfunção dinâmica dependerá do grau de perturbação e do grau de insuficiência funcional das sinapses inibitórias (Glass e McKey, 1988).

Nicoll e colaboradores (1990) propuseram uma subdivisão topográfica e funcional das populações de neurônios do SNC. A maioria dos neurônios do SNC forma um subsistema denominado informacional ou hierárquico (por ser o mais numeroso). São fibras corticais caracterizadas por fibras de condução rápida (mielínicas), dotadas de atividade excitatória (mediadas por glutamato e aspartato) e inibitória (mediadas por glicina e GABA) específicas, predominantemente centrípetas (irradiam-se do córtex para estruturas subcorticais). Um segundo subsistema de neurônios, chamado inespecífico ou modulador, de origem subcortical, caracteriza-se por fibras de condução lenta (amielínicas), predominantemente centrífugas (irradiam-se dos núcleos subcorticais para o córtex). Esse subsistema recebe o nome de inespecífico porque suas propriedades de excitação ou inibição podem variar segundo a região do SNC e segundo o estado funcional da sinapse. Ele também compartilha monoaminas com o sistema nervoso vegetativo (adrenalina, noradrenalina, serotonina, acetilcolina, dopamina), além de muitos outros neurotransmissores.

Os substratos cortical e subcortical dialogam através destas fibras, que interagem formando uma complexa rede regulada por um dinamismo extraordinário de feedbacks negativos, ora predominando o subsistema hierárquico 
excitatório, ora o hierárquico inibitório, ora o modulador excitatório, ora o modulador inibitório (Toro, Talavera e Guillén, 1997). Este modelo simplificado confirma o que já foi antevisto, por Pavlov, como um dinamismo de excitações e inibições, a base funcional da atividade nervosa superior.

Toro, Talavera e Guillén (ibid.) propuseram um modelo dinâmico para estas interações sujeitas a instabilidades. Este funcionamento operado por fluxos está naturalmente sujeito a flutuações, e as quatro variedades funcionais interagem com suas atividades oscilando segundo o nível de estimulação numa dada área do SNC. Uma perturbação, externa ou interna, que aumente a atividade numa dada região do SNC, altera também o padrão de interação entre as quatro variedades funcionais. Se a perturbação é significativa, uma destas variedades funcionais pode experimentar uma sobrecarga funcional e pode instalar-se aí um feedback positivo que ampliará a perturbação até atingir uma bifurcação, originando uma zona de flutuação local formada por uma população de neurônios hierárquicos e difusos, com organização e nível de estimulação distintos do resto do sistema que não experimentou flutuação, entrando em competição com este.

Esta zona de flutuação torna-se autônoma, ou seja, passa a operar em paralelo assemelhando-se a um parasita do SNC. Vinogradov e colaboradores (1992) propuseram o termo "parasitismo dinâmico" para enfatizar o caráter estável e autônomo destas zonas de flutuação, melhor designadas como um "circuito autônomo de neurônios acoplados" (CAA).

Podemos considerar dois tipos de CAA: (1) aqueles que são flutuações reversíveis, ou seja, que não atingiram o limiar crítico (ponto de bifurcação) e podem ser corrigidas pela parte contígua que não experimentou flutuação; e (2) aqueles que atingiram uma bifurcação e, portanto, se auto-organizaram em complexos funcionais autônomos, para os quais o sistema que os engloba tentará um reajuste compensatório. Neste caso, a compensação não corrige a alteração e dá origem a uma disfunção do SNC, referida como homeostase patológica (King e Liston, 1990), ou doença dinâmica (Goldberger e col., 1990), ou desmantelamento da homeostase (Pires, 1981). No primeiro caso, teríamos os transtornos transitórios, como os buffeés delirantes, alterações psicóticas transitórias durante um período de estresse etc.; e, no segundo caso, as epilepsias, as descompensações psicossomáticas, tais como a hipertensão arterial essencial e outras alterações funcionais, depressão, certas formas de delírios e alucinações, doença do pânico, estados obsessivos-compulsivos, complexos dissociativos, etc.

A região contígua aos CAA tenta compensar este inibindo sua irradiação de modo a encapsular o distúrbio, e isto pode ocorrer inclusive entre os substratos cortical e subcortical. Esses componentes inibidos também participam da formação dos sintomas (deficitários) comumente vistos ao lado dos sintomas produtivos regidos pelo CAA. 
Este modelo dinâmico atualiza a psiquiatria pavloviana, que formula uma base dialética no funcionamento cerebral para explicar a origem da maioria dos transtornos mentais e do comportamento. Pavlov já havia percebido estas alterações dinâmicas locais, e denominou os CAAs de "focos corticais patológicos" ou "focos estacionários inertes". Não é incompatível que a psicanálise possa revisar algumas de suas teorias e se beneficiar deste modelo.

Os CAAs não são mais estruturas teóricas, e têm sido comprovados em estudos de PET-scan como áreas hiperativadas (focos excitados inertes) ou hipoativadas (focos inibidos inertes) em certos transtornos mentais.

\section{Dinâmica do tratamento psiquiátrico}

Quando o CAA é transitório, ou seja, ainda não se auto-organizou, uma medicação específica ou psicoterapia breve podem levar à dissolução do mesmo. Uma medicação específica, atuando sobre neurotransmissores, pode reforçar os mecanismos de correção da zona de flutuação por meio das áreas contíguas que apresentem um bom grau de conectividade com o CAA. Muitas vezes essa remissão é espontânea, ao cessar o estressor ou a alteração fisiológica (exemplo, um distúrbio endócrino) que vulnerabilizava o eixo cortico-subcortical. A psicoterapia aqui seria de ajuda já que permite ao indivíduo conhecer os fatores que desencadeiam suas "crises" e, assim, obter sua cooperação.

Os CAA auto-organizados dificilmente poderão ser dissolvidos já que, sendo autônomos, operam em paralelo e independente do fluxo da atividade nervosa superior. Alterações da maturação do sistema nervoso ou da conectividade em certas áreas podem concorrer para a existência de zonas de flutuação autoorganizadas. Estes seriam responsáveis pelos transtornos mentais maiores, cujos tratamentos mais usados são a psicofarmacoterapia, a eletroconvulsoterapia (ECT) e, em menor proporção, a indução ou supressão do sono.

A psiquiatria moderna baseia-se no raciocínio reducionista de que a psicopatologia associa-se a mudanças neuroquímicas nas sinapses. Isto fundamenta o tratamento psicofarmacológico que, teoricamente, deve reverter ou compensar as mudanças nos níveis de transmissores ou seus receptores. A abordagem dinâmica não é contrária a este raciocínio, mas amplia a questão para o nível dos subsistemas autonomizados (Toro, Talavera e Guillén, 1997), hoje reconhecidos como alvos prioritários no desenho de novos tratamentos. Isto levou ao aparecimento dos antipsicóticos atípicos e novos antidepressivos, onde não apenas o neurotransmissor envolvido, mas também a via nervosa implicada são os alvos da ação terapêutica. 
Temos então que considerar o tratamento sob o ponto de vista de uma mudança no nível de ativação, partindo do princípio de que uma perturbação pode vir a corrigir uma homeostase patológica se for corretamente aplicada (Yang, Ding e Mandell, 1995; Guevara et al., 1981). Isso significa que é possível inibir uma zona de flutuação ativa, ou ativar seletivamente áreas cerebrais hipoativas para entrar em equilíbrio compensatório com o CAA. Esta nova classe de psicofármacos não atua globalmente, e sim seletivamente em vias supostamente envolvidas na formação dos sintomas psicóticos, onde estariam, justamente, os CAAs. É possível, inclusive, que a latência inicial dos antidepressivos e antipsicóticos se deva a uma resistência inicial da homeostase patológica à ação dos fármacos (Hyman e Nestler, 1996).

A ECT pressupõe uma normalização generalizada dos padrões de interação entre os subsistemas, inicialmente por uma estimulação brusca seguida de uma inibição global. Isto tem dado resultado em situações em que o transtorno é hiperativo e não responde a nenhuma outra forma de intervenção. À medida que a tecnologia do ECT se aperfeiçoa, como ocorreu com os desfibriladores, tornase possível estabelecer protocolos mais eficazes e mais seletivos. Também é possível que a psicocirurgia venha se aperfeiçoar em técnicas capazes de eliminar seletivamente a população de fibras que conformam um CAA.

O sono já foi muito usado, na psiquiatria de orientação pavloviana, como terapia nos processos em que ocorriam grandes estresses emocionais, inibindo o desenvolvimento de transtornos de ansiedade. O sono amortece flutuações incipientes restaurando o equilíbrio original, e isto parece decorrer de uma alternância entre inibição e excitação, o que ajudaria a regular o tônus nervoso. Sabe-se que o sono lento é inibitório, associado a um aumento de catecolamina/ serotonina, enquanto o sono REM, que se alterna com aquele, é excitatório, com predomínio colinérgico. Esta oscilação ativatória pode neutralizar ou reduzir o efeito de determinados CAAs; porém, em alguns casos, pode favorecê-lo, o que explica porque a supressão seletiva do sono algumas vezes melhora a depressão (Liebenluft e Wehr, 1992; Bauer et al., 1995) e ansiedade (Silvestri e Root, 2008). $\mathrm{O}$ uso da estimulação magneto-transcraniana tem sido promissor no tratamento da depressão e manias e sua ação reforça o modelo apresentado neste trabalho (Toro, Montes e Talavera, 2001)

Devemos aqui considerar igualmente que uma abordagem psicoterapêtica pode ser de significativo valor na redução do aporte de perturbação externa ao sistema nervoso. Também não é impossível que auxilie no amortecimento da atividade de um CAA. Isto se torna mais objetivo quando eliminamos o dualismo mente-corpo e abordamos o ser humano como um todo integrado, inseparável do meio e sociedade em que vive, readaptando-se constantemente ao seu entorno, 
não estando unicamente à mercê da sua biologia e cultura, tendo liberdade suficiente para, em certa medida, modificar sua fisiologia e sua orientação psíquica.

É por esta unidade indissolúvel que certos fármacos têm mais valor psicoterapêutico no contexto da relação médico-paciente do que farmacológico, e certas intervenções psicoterapêuticas são mais especificamente psicobiológicas do que psíquicas.

Em resumo, as zonas de flutuação são vias quimioelétricas interagentes operando de modo paralelo e parasita, obrigando o SNC a ajustar-se compensatoriamente. Estas zonas de flutuação podem distorcer, irreversivelmente, o funcionamento cerebral, aumentar sua vulnerabilidade para novas recorrências e limitar a resposta terapêutica, e isto não está apenas restrito às síndromes psicóticas (Angst et al., 1996). Concorrem para isto a ação cooperativa entre os estressores e a vulnerabilidade do sistema nervoso que, juntas, mantêém determinadas combinações de percepções, afetos, cognições e comportamentos que formam o que denominamos de transtornos mentais. O tratamento ideal será aquele que auxilia o SNC, de forma mais rápida e eficaz, a compensar a insuficiência funcional relativa na interação entre o circuito afetado e o seu entorno reativo não afetado.

\section{Conclusão}

A psiquiatria moderna adotou a postura reducionista de associar psicopatologia à fisiologia sináptica. Isto produziu avanços importantes na farmacoterapia dos transtornos mentais. Entretanto, a introdução de princípios da dinâmica não-linear em psiquiatria nos fez avançar para uma abordagem mais sistêmica, focalizando vias e áreas específicas do cérebro como os substratos psicopatológicos. Esta tendência, iniciada nos anos 1990 (Freeman, 1992; Mandell e Selz, 1992; Globus e Arpaia, 1994), tem agora produzido seus resultados, especialmente na concepção de psicofarmacoterapia e psicoterapia.

\section{Referências}

Angst, J.; Kupfer, D.J.; Rosenbaum, J.F. Recovery from depression: risk and reality. Acta Psychiatrica Scandinavica, Singapura, n. 93, p. 413-19, 1996.

BAUER, J. et al. Induction of cytokine synthesis and fever suppresses REM sleep and 
improves mood in patients with major depression. Biological Psychiatry, Filadélfia, n. 38, p. 611-21, 1995.

Freeman, W. Chaos in psychiatry. Biol. Psychiatry, Filadélfia, n. 31, p. 1079-81, 1992.

Glass, L.; Mackey, M.C. From clocks to chaos. The rhythms of life. Princeton: Princeton University Press, 1988.

Globus, G.G.; Arpaia, J.P. Psychiatry and the New Dynamics. Biol. Psychiatry, Filadélfia, n. 35, p. 352-64, 1994.

Goldberger, A.L. Nonlinear dynamics, fractals and chaos: Applications to cardiac electrophysiology. Ann. Biomed. Eng., Heilderberger, n. 18, p. 195-98, 1990.

. Is the normal heartbeat chaotic or homeostatic? News Physiol. Sci., Bethesda, MA, n. 6, p. 87-91, 1991.

Guevara, M.R.; Glass, L.; Shrier, A. Phase locking, period-doubling bifurcations and irregular dynamics in periodic stimulated cardiac cells. Science, Washington, n. 214, p. 1350-2, 1981.

Hyman, S.; Nestler, E. Initiation and adaptation: A paradigm for understanding psychotropic drug action. American Journal of Psychiatry, Arlington, VA, n. 153, p. 151-62, 1996.

King, B.H.; Liston, E.H. Proposal for the mechanism of action of convulsive therapy: a synthesis. Biol. Psychiatry, Filadélfia, n. 27, p. 76-94, 1990.

Mackey, M.C.; Mitton, J.G. Dynamical diseases. Ann. N.Y. Acad. Sci., New York, n. 504, p. 16-32, 1987.

Mandell, A.J. Nonlinear dynamics in brain processes. Psychopharmacol. Bull., Sta. Monica, CA, n. 18, p. 59-63, 1982.

Mandell, A.Y.; Selz, K.A. Dynamical systems in psychiatry: Now what? Biol. Psychiatry, Filadélfia, n. 32, p. 299-301, 1992.

Milsum, J.H (ed.). Positive feedback: a general systems approach to positive/negative feedback and mutual causality. Oxford: Pergamon Press, 1968.

Nicoll, R.; Malenka, R.; Kaver, J. Functional comparison of neurotransmitter receptor subtypes in mammalian central nervous system. Physiological Rev., Bethesda, MA, n. 70, p. 513-65, 1990.

Paulus, M.P.; BrafF, D.L. Chaos and schizophrenia: Does the method fit the madness? Biol. Psychiatry, Filadélfia, n. 53, p. 3-11, 2003.

Peterson, B.S.; Leckman, J.F. The temporal dynamics of tics in Gilles de la Tourette Síndrome. Biol. Psychiatry, Filadélfia, n. 44, p. 1337-48, 1998.

Pezard, L. et al. Depression as a dynamical disease. Biol. Psychiatry, Filadélfia, n. 39, p. 991-9, 1996. 
Pires, N. Sentir-se doente (o desmantelamento homeostático na clínica prática). Rio de Janeiro: Gráfica Olímpica Editora, 1981.

Poot, R. Is healthy to be chaotic? Science, Washington, n. 243, p. 604-7, 1989.

Prigogine, I.; Stengers, I. A nova aliança. Brasília: Universidade de Brasília, 1984a. ; __. Order out of chaos. London: Bantham, 1984b.

Redington, D.J.; ReidBord, S.P. Chaotic dynamics in autonomic nervous system activity of a patient during a psychotherapy session. Biological Psychiatry, Filadélfia, n. 31, p. 993-1007, 1992.

SCHIFF, S.J et al. Controlling chaos in the brain. Nature, Londres, n. 370, p. 615-20, 1994.

SilvestRI, A.J.; Root, D.H. Effects of REM deprivation and an NMDA agonist on the extinction of conditioned fear. Physiology and Behavior, Filadélfia, n. 93, p. 274-81, 2008.

Skarda, C.A.; Freeman, W.J. Chaos and the new science of the brain. Concepts in Neuroscience, Singapura, v. 1, n. 2, p. 275-85, 1990.

Toro, M.G.; Talavera, J.A.; Guillén, A.G. Teoria de sistemas dinámico no lineares y trastorno mentales. Psicología.com [On-line], v. 1, n. 1, 48 parágrafos, 1997. Disponível em http://www.psiquiatria.com/vollnum1/art_6.htm. Acesso em 2.11.2005.

Toro, M.G.; Montes, J.M.; Talavera, J.A. Functional cerebral asymetry in affective disorders: new facts contributed by transcranial magnetic stimulation. Journal of Affective Disorders, Filadélfia, n. 66, p. 103-9, 2001.

Vinogradov, S.; Xing, R.J.; Huberman, B.A. An associationist model of the paranoid process: Application of phase transitions in spreading activation networks. Psychiatry, Massachusetts, n. 55, p. 79-94, 1992.

Von Bertalanffy, L. Teoria geral dos sistemas. 2. ed. Petrópolis: Vozes, 1975.

Yang, W.; Ding, W.; Mandell, A.J. Preserving chaos: Control strategies to preserve complex dynamics with potential relevance to biological disorders. Physical Review E, Filadélfia, n. 51, p. 102-10, 1995.

YeRAGANI, V.K. et al. Diminished chaos of heart rate time series in patients with major depression. Biol. Psychiatry, Filadélfia, n. 51, p. 733-44, 2002.

\section{Resumos}

(Dinámica no linear y psiquiatría: la naturaleza dinámica de las enfermedades mentales)

La psiquiatría moderna adopto la postura reduccionista de asociar psicopatología a la fisiología sináptica. Ese hecho produjo avances importantes en la

Rev. Latinoam. Psicopat. Fund., São Paulo, v. 11, n. 1, p. 105-118, março 2008 
farmacoterapia de los trastornos mentales. De todos modos, la introducción de principios de la dinámica no linear promovió un cambio del enfoque sináptico para el enfoque de las vías específicas patológicamente autónomas envueltas en los fenómenos psicopatológicos. Esta tendencia tuvo inicio en los años 90 y trajo un concepto más sistémico de fisiología y de tratamiento en psiquiatría.

Palabras claves: Psiquiatría e dinámica no linear, termodinámica del no equilibrio e psiquiatría, enfermedades dinámicas

(La dynamique non linéaire et la psychiatrie: la nature dynamique des maladies mentales)

La psychiatrie moderne a adopté une position réductionniste qui associe la psychopathologie à la physiologie synaptique, ce qui se trouve à l'origine d'importants progrès en pharmacothérapie des troubles mentaux. Cependant, l'introduction de principes de la dynamique non linéaire a provoqué un déplacement de l'accent synaptique vers l'accent sur les voies spécifiques pathologiquement autonomes impliquées dans les phénomènes psychopathologiques. Cette tendance a surgit au début des années 90 et a apporté un concept plus systémique de la physiopathologie et du traitement en psychiatrie.

Mots clés: Psychiatrie et dynamique non-linéaire, thermodynamique du non-equilibre et psychiatrie, maladies dynamiques

(Nonlinear dynamics and psychiatry: the dynamic nature of mental disorders)

Modern psychiatry was built on the association between psychopathology and synaptic physiology. This brought about important advances in the pharmacotherapy of mental illness. However, the introduction of the principles of nonlinear dynamics changed the focus on synaptic aspects into a focus on specific and pathologically autonomous approaches involved in psychopathologal phenomena. This trend began in the 1990s and brought in more systemic concepts of physiopathology and psychiatric treatment.

Key words: Psychiatry and nonlinear dynamics, psychiatry and non-equilibrium thermodynamics, dynamic diseases

Versão inicial recebida em janeiro de 2008 Versão aprovada para publicação em fevereiro de 2008

\section{Fernando Portela Câmara}

MD, PhD; professor associado, SEDI-IMPPG-Universidade Federal do Rio de Janeiro (Rio de Janeiro, RJ, Brasil)

Rua Pinheiro Machado, 25/405

22231-090 Rio de Janeiro, RJ, Brasil

e-mail: fp3camara@yahoo.com.b

Rev. Latinoam. Psicopat. Fund., São Paulo, v. 11, n. 1, p. 105-118, março 2008 